\title{
The role of potassium channels in the mechanism of vasodilatation of human umbilical vein induced by resveratrol
}

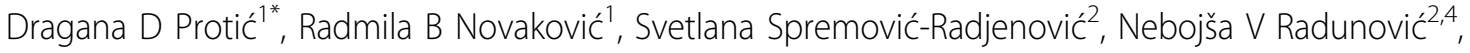 \\ Helmut Heinle ${ }^{3}$, Vladimir I Kanjuh ${ }^{4}$, Ljiljana C Gojković-Bukarica ${ }^{1}$ \\ From 18th Scientific Symposium of the Austrian Pharmacological Society (APHAR). Joint meeting with the \\ Croatian, Serbian and Slovenian Pharmacological Societies. \\ Graz, Austria. 20-21 September 2012
}

\section{Background}

Resveratrol (RSV) is polyphenol present in various kinds of food which we consume on daily basis. In the last ten years there has been growing importance of RSV in the literature. It is well known that RSV has many different beneficial effects on human health. RSV is partly responsible for the cardiovascular benefits of red wine. However, the mechanism of vasodilatation induced by RSV is unclear. There are many target molecules of RSV which could play an important role in the mechanism of action of RSV. The aim of our study was to define the role of $\mathrm{K}^{+}$ channels in the RSV-induced vasodilatation of human umbilical vein (HUV) denuded of endothelium.

\section{Methods}

HUV rings were precontracted with serotonin (5-HT) or with $100 \mathrm{mM} \mathrm{K}^{+}$. Concentration-response curves were obtained by adding increasing concentrations of RSV, from 1 to $100 \mu \mathrm{M}$. In order to test the role of vascular $\mathrm{K}^{+}$ channels in this vasorelaxation, various $\mathrm{K}^{+}$channels blockers were added to the organ bath 20 minutes before RSV.

\section{Results}

RSV induced concentration-dependent vasodilatation $\left(\mathrm{EC}_{50}=16.5 \mu \mathrm{M}\right)$. A selective blocker of ATP-sensitive $\mathrm{K}^{+}$ channels, glibenclamide $(10 \mathrm{mM})$, induced a significant shift to the right $(\mathrm{p}<0.05)$ of the concentration-response curve for RSV $\left(\mathrm{EC}_{50}=38.0 \mu \mathrm{M}\right)$. 4-Aminopyridine (4-AP, $1 \mathrm{mM})$, a blocker of voltage-gated $\mathrm{K}^{+}$channels, also

\footnotetext{
* Correspondence: draganap@med.bg.ac.rs

${ }^{1}$ Institute of Pharmacology, Clinical Pharmacology and Toxicology, Medical Faculty, University of Belgrade, Serbia

Full list of author information is available at the end of the article
}

induced a significant shift to the right $\left(\mathrm{EC}_{50}=49.0 \mu \mathrm{M}\right.$, $\mathrm{p}<0.05$ ). Tetraethylamonium (TEA, $10 \mathrm{mM}$ ), which predominantly inhibits large conductance $\mathrm{Ca}^{2+}$-activated $\mathrm{K}^{+}$ channels, and barium chloride $\left(\mathrm{BaCl}_{2}, 1 \mathrm{mM}\right)$, which blocks inward rectifier $\mathrm{K}^{+}$channels, antagonized the response to RSV $\left(\mathrm{EC}_{50}=28.0 \mu \mathrm{M}, \mathrm{p}<0.05\right.$ and $\mathrm{EC}_{50}=$ $50 \mu \mathrm{M}, \mathrm{p}<0.05$, respectively). Concentrations of RSV above $10 \mu \mathrm{M}$ relaxed HUV rings bathed in a medium containing $100 \mathrm{mM} \mathrm{K}^{+}\left(\mathrm{EC}_{50}=47 \mu \mathrm{M}, \mathrm{p}<0.05\right)$.

\section{Conclusions}

These results suggest that RSV induces endotheliumindependent vasorelaxation of HUV. $\mathrm{K}^{+}$channels are involved in the vasodilatation of HUV induced by RSV, when RSV is applied in concentrations up to $10 \mu \mathrm{M}$. However, it seems that RSV has an additional, $\mathrm{K}^{+}$channel-independent mechanism of action when applied in concentrations higher than $10 \mu \mathrm{M}$.

\section{Author details \\ ${ }^{1}$ Institute of Pharmacology, Clinical Pharmacology and Toxicology, Medical Faculty, University of Belgrade, Serbia. ${ }^{2}$ Institute of Gynecology and Obstetrics, Clinical Center of Serbia, 11129 Belgrade, Serbia. ${ }^{3}$ Institute of Physiology, University of Tübingen, 72076 Tübingen, Germany. \\ ${ }^{4}$ Serbian Academy of Sciences and Arts, 11129 Belgrade, Serbia.}

Published: 17 September 2012

doi:10.1186/2050-6511-13-S1-A29

Cite this article as: Protić et al:: The role of potassium channels in the mechanism of vasodilatation of human umbilical vein induced by resveratrol. BMC Pharmacology and Toxicology 2012 13(Suppl 1):A29. 